\title{
ADAPTIVE TRILATERAL FILTER FOR IN-LOOP FILTERING
}

\author{
Akitha Kesireddy and Mohamed El-Sharkawy \\ Purdue School of Engineering and Technology \\ melshark@iupui.edu
}

\begin{abstract}
High Efficiency Video Coding (HEVC) has achieved significant coding efficiency improvement beyond existing video coding standard by employing several new coding tools. Deblocking Filter, Sample Adaptive Offset (SAO) and Adaptive Loop Filter (ALF) for in-loop filtering are currently introduced for the HEVC standard. However, these filters are implemented in spatial domain despite the fact of temporal correlation within video sequences. To reduce the artifacts and better align object boundaries in video, a proposed algorithm in in-loop filtering is proposed. The proposed algorithm is implemented in HM-11.0 software. This proposed algorithm allows an average bitrate reduction of about $0.7 \%$ and improves the PSNR of the decoded frame by $0.05 \%, 0.30 \%$ and $0.35 \%$ in luminance and chroma.
\end{abstract}

\section{KEYWORDS}

HEVC, Trilateral Filter and In-Loop Filter.

\section{INTRODUCTION}

HEVC is the new video coding standard developed by the joint collaboration of the ITU-T Video Coding Experts Group (VCEG) and the ISO/IEC Moving Picture Experts Group (MPEG). The main aim of the HEVC is improving the compression efficiency of the H.264/AVC standard by almost 50\% and maintaining the same computational complexity. Many coding tools are included to reduce the distortion between the original frames and decoded frames produced by the lossy coding.

The name loop filtering reflects that the filtering is done by removing the blocking artifacts [1].H.264/AVC includes an in-loop Deblocking filter. HEVC employs a Deblocking filter similar to the one used in H.264/AVC but expands an in-loop processing by introducing two new tools: $\mathrm{SAO}$ and ALF. These techniques are implemented to reduce the distortion introduced by the encoding process(prediction, transform, and quantization). By including these filtering techniques, the pictures will serve as better references for motion- compensated prediction since they have less encoding distortion.

Over the past several years many algorithms have been proposed for reducing the blocking artifacts and the bit rate [2] - [5]. These algorithms can be categories into three types: first type is a post processing algorithm for removing blocking artifacts for highly compressed images in the DCT domain [2], second one reduces the blocking artifacts carried out at encoding schemes and third one reduces the temporal redundancy of ALF parameters by reusing the prior transmitted filter parameters [3]. In [4], a strong filter is selectively applied to blocks having small artifacts to

Natarajan Meghanathan et al. (Eds) : ICAIT, ICDIPV, ITCSE, NC - 2014

pp. 35-41, 2014. (C) CS \& IT-CSCP 2014

DOI : $10.5121 /$ csit.2014.4705 
avoid harmful side effect of filtering. A weak filter is applied to the other blocks to slightly correct them. In [5],an adaptive in-loop bilateral filter selecting the optimal filter parameters, based on the image characteristics, is proposed to minimise the Lagrangian Rate-Distortion.

In this paper, we propose an algorithm to reduce the bit rate and improve the video quality by combining a trilateral filter and adaptive filter together, evaluate the effect of proposed algorithm on the quality of the output and compare their results to the evaluated results of the original algorithm of HEVC for various quantization parameters.

The rest of the paperis organized as follows: Section II describes the adaptive loop filtering. Section III describes the trilateral filter. Section IV describes the proposed algorithm. Finally, the experimental results and conclusion are shown in sections V and VI.

\section{HEVC ADAPTIVE LOOP FILTERING}

This section describes ALF core techniques employed in the final ALF version in HM-11.0. The filter includes Wiener filter, filter shapes, and coefficient coding. The Wiener filter minimizes the mean square error between the desired samples and the filtered samples. The desired samples are the original picture. The to-be-filtered samples are the output picture of SAO. The filtered samples are the output picture of ALF.

In [1], an ALF is applied to the reconstructed signal after the de-blocking filter and SAO. The filter is adaptive in the sense that the coefficients are signalled in the bit stream and can therefore be designed based on image content and distortion of the reconstructed picture. The filter is used to restore the reconstructed picture such that the mean-squared error between the source picture and the reconstructed picture is minimized.

Test Model HM-3.0 uses a single filter shape which is a cross overlaid on a 3 x 3 square with nine coefficients, to be encoded in the bit stream. The number of taps in the filter is greater than nine due to symmetry; however, every picture may not need all the nine taps as in HM-7.0 [6]. This test model has different filter square shapes $5 \times 5,7 X 7$ and $9 \times 9$, where these shapes can be selected for different pictures. In HM-6.0 and HM-7.0, number of coefficients is reduced to half by changing the ALF from square to diamond shape. The combination of 9X7-tap cross shape and 3X3-tap rectangular shape generates the filter shape of ALF in HM-7.0.The Filter coefficients are derived by solving Wiener-Hopf equation [6].ALF in HM7.0 reduces the number of coefficient by half which in turn reduces the number of multiplications by half, which significantly reduces the chip area for ALF.

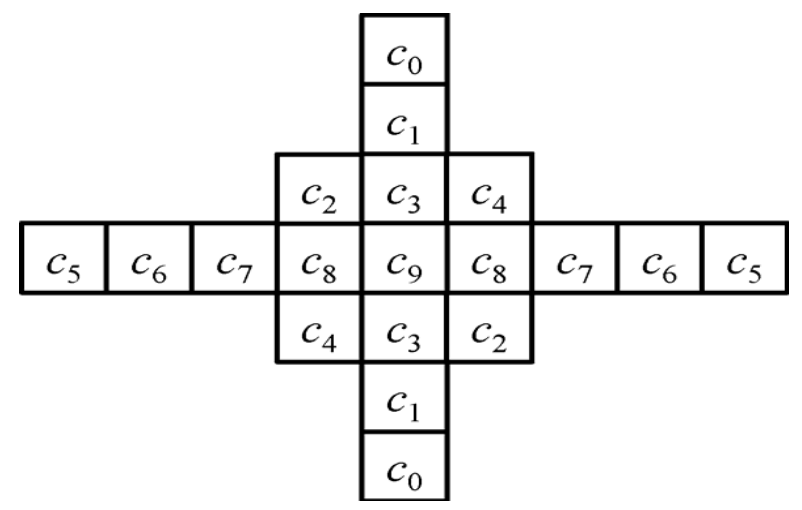

Figure 1: Filter shape of ALF in HM-6.0 and HM-7.0. 


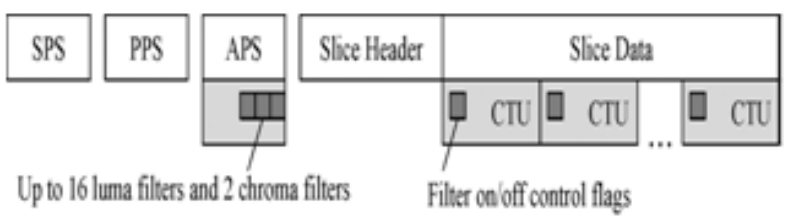

Figure 2: Locations of ALF parameters in the bit stream.

\section{Syntax Design}

There are two types of coded information for ALF: filter coefficient parameters and filter on/off control flags. As shown in Fig. 2, the filter coefficient parameters are located in a picture-level header called APS, and the filter on/off control flags are interleaved in slice data with CTUs. The filter coefficient parameters include picture-level on/off control flags for three color components, number of luma filters (i.e., class/region merging syntax elements for BA/RA), and corresponding filter coefficients. Up to 16 luma filters, one $\mathrm{Cb}$ filter, and one $\mathrm{Cr}$ filter per picture can be signalled. Filter on/off control flags are used to provide better local adaptation. In addition to the picture-level filter on/off control flags in APS, there are also slice-level and CTU-level filter on/off control flags. In slice header, similarly, filter on/off control flags for three color components are coded. The signalling of slice-level filter on/off control flags can solve a slice parsing problem when the referenced APS of the slice is lost [7]. If the slice-level on/off control flag indicates ALF-on, CTU-level filter on/off control flags are interleaved in slice data and coded with CTUs; otherwise, no additional CTU-level filter on/off control flags are coded and all CTUs of the slice are inferred as ALF-off.

APS was removed from HEVC standard after HM-8.0.

As APS is removed the related syntax elements of filter parameters in slice header ALF is implemented.

\section{Trilateral Filter}

An image is defined by $\mathrm{f}(\mathrm{x}) \in R^{n}(\mathrm{n}=$ dimensionality), where $\mathrm{x} \in \Omega$ is the pixel position in image domain $\Omega$. Generally speaking, an $n$-D (n-dimensional) pixel-discrete image has an image domain defined as, $\varnothing \subset \Omega \subseteq X_{n} \subset N^{n}(X n$ is our maximum discrete index set of the image domain in dimension $n$ ). A smoothing operator will reduce an image to a smoothed version of itself, specifically $\mathrm{S}(f)=\mathrm{s}$, where $\mathrm{s}$ is in the same image domain as $f$. To introduce the trilateral filter, we must first define the bilateral [8] case; we will then go on to define the traditional trilateral filter using this notation.

The trilateral filter is a "gradient-preserving" filter [8]. It aims at applying a bilateral filter on the current plane of the image signal. The trilateral case only requires the specification of one parameter $\sigma_{1}$. At first, a bilateral filter is applied on the derivatives of $f$ (i.e., the gradients):

$$
\begin{gathered}
g_{f}(x)=\frac{1}{k_{\nabla}} \int_{\Omega} \nabla f(x+\mathbf{a}) \cdot w_{1}(\mathbf{a}) \cdot w_{2}(\|\nabla f(x+\mathbf{a})-\nabla f(x)\|) d \mathbf{a} \\
k_{\nabla}(x)=\int_{\Omega} w_{1}(\mathbf{a}) \cdot w_{2}(\|\nabla f(x+\mathbf{a})-\nabla f(x)\|) d \mathbf{a}
\end{gathered}
$$

To approximate $\nabla f(x)$, forward differences are used, and more advanced techniques (e.g., Sobel gradients, 5-point stencil) are left for future studies. For the subsequent second bilateral filter, suggested the use of the smoothed gradient $g_{f}(x)$ [instead of $\nabla f(x)$ for estimating an approximating plane 


$$
p_{f}(x, \mathbf{a})=f(x)+g_{f}(x) \cdot \mathbf{a}
$$

Let $f_{\nabla}(x, \mathbf{a})=f(x+\mathbf{a})-p_{f}(x, \mathbf{a})$. Furthermore, a neighbour-hood function

$$
N(x+\mathbf{a})=\left\{\begin{array}{lr}
1 & \text { if }\left|g_{f}(x+\mathbf{a})-g_{f}(x)\right|<c \\
0 & \text { otherwise }
\end{array}\right.
$$

is used for the second weighting. Parameter $\mathrm{c}$ specifies the adaptive region and is discussed further below. Finally,

$$
\begin{gathered}
s(x)=f(x)+\frac{1}{k_{\nabla}(x)} \int_{\Omega} \nabla f(x, \mathbf{a}) \cdot w_{1}(\mathbf{a}) \cdot w_{2}(\nabla f(x, \mathbf{a})) \cdot \mathrm{N}(x, \mathbf{a}) d \mathbf{a} \\
k_{\nabla}(x)=\int_{\Omega} w_{1}(\mathbf{a}) \cdot w_{2}(\nabla f(x, \mathbf{a})) \cdot \mathrm{N}(x, \mathbf{a}) d \mathbf{a}
\end{gathered}
$$

The smoothed function $s$ equals $S_{T L}(f)$.

Again, $w_{1}$ and $w_{2}$ are assumed to be Gaussian functions, with standard deviations $\sigma_{1}$ and $\sigma_{2}$, respectively. The method requires specification of parameter $\sigma_{1}$ only, which is at first used to be the diameter of circular neighbour-hoods at $x$ in $f$; let $\overline{g_{f}}(x)$ be the mean gradient of $f$ in such a neighbourhood. The parameter for $w_{2}$ is defined as follows:

$\left(\beta=0: 15\right.$ was chosen). Finally, $c=\sigma_{2}$.

$$
\sigma_{2}=\beta \cdot\left|\max _{x \in \Omega} \overline{g_{f}}(x)-\min _{x \in \Omega} \overline{g_{f}}(x)\right|
$$

\section{Proposed Algorithm}

There are three in-loop filtering techniques in HEVC; namely, the de-blocking filtering, the Sample Adaptive Offset (SAO) and the Adaptive Loop Filter (ALF). After the details of these filters in the previous sections, we design the proposed filter in in-loop filtering process.

\section{Boundary Block Detection}

Trilateral filter works in the context of block-based processing. The trilateral filter might introduce other blocking artifacts if it is applied to all the blocks in a frame, so it is only applied to blocks in object boundaries. This is called region-based filtering. The standard deviation of the block is used to detect where the boundary block. Non-boundary blocks usually consist of homogeneous pixel values and have a smaller variance. When the standard deviation of a block exceeds a pre-defined value, the trilateral filtering is performed and the standard deviation for an NXN block is:

$$
\operatorname{STD}=\operatorname{Sqrt}\left\{\frac{1}{\mathrm{~N} \times \mathrm{N}} \sum_{\mathrm{i}=0}^{\mathrm{N}-1} \sum_{\mathrm{j}=0}^{\mathrm{N}-1}\left[\mathrm{I}(\mathrm{i}, \mathrm{j})-\mathrm{Mean}_{\mathrm{blk}}\right]^{2}\right\}
$$

Where $\mathrm{N}$ is the block size, $\mathrm{I}(\mathrm{i}, \mathrm{j})$ is the pixel intensity, and Mean is the mean of the block.

\section{In-Loop Filtering}

After the details of ALF and trilateral filter in SECTION II, it is now essential to define how to combine these filters in the HEVC in-loop filtering process. As described in the introduction. The trilateral filter is "gradient-preserving" filter suited to remove the blocking artifacts whereas the Adaptive loop filter is more targeted to reduce the bit-rate. Therefore, it is appropriate to combine these two filters by selecting, for each image block in the reconstructed frame. This is the main idea behind the proposed algorithm whose processing considered each block along with the deblocking filter. The filtering reduces the bit-rate and improves the PSNR values and is not 
complicated when compared with other algorithms. Now the procedure is detailed in steps by supposing a input frame $\mathrm{F}$ into the in-loop filter. The performed steps are:

1. Partition $\mathrm{F}$ into block size of $\mathrm{B}$

2. Using the standard deviation of a block detect the object boundaries

3. Over the object boundary perform TLF to obtain $\mathrm{F}^{\mathrm{TLF}}$

4. Perform DBF over the remaining blocks of the frame to obtain $\mathrm{F}^{\mathrm{DBF}}$

5. Finally the combined frame $\mathrm{F}^{\mathrm{TDBF}}$ is obtained

6. Over the whole frame $\mathrm{F}^{\mathrm{TDBF}}$ perform ALF to obtainF ${ }^{\mathrm{TDALF}}$.

In this algorithm by considering the region characteristics of the block only the block boundaries are filtered by trilateral filter. Therefore, we adopt the quad-tree structure of LCU in HEVC. For every CUs in LCU, we check whether its standard deviation is above a certain threshold. If the condition is met, we perform the trilateral filtering in this block. Later ALF is performed over the whole frame. The overall flow chart of the proposed in-loop filter for HEVC is shown in Figure 3

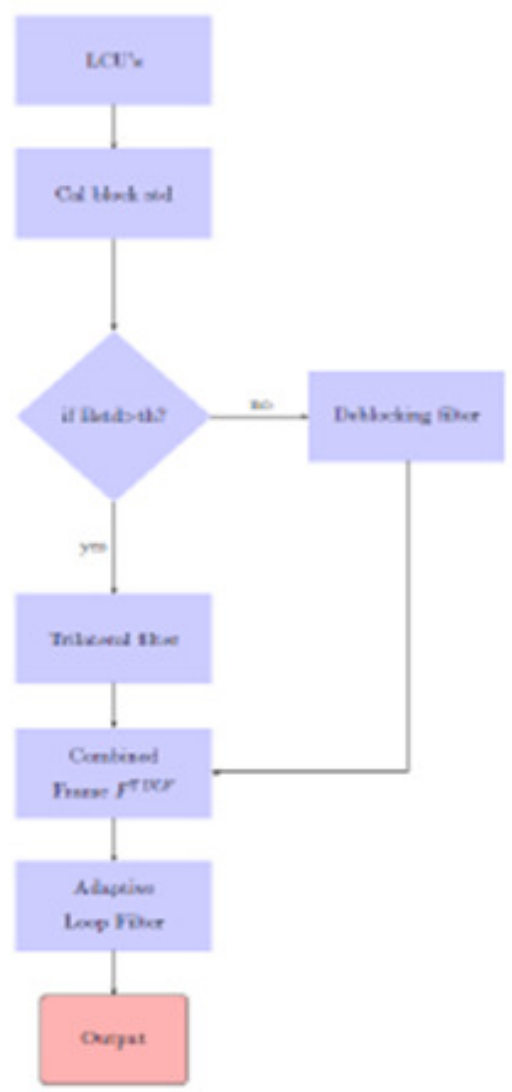

Figure 3: Flow chart of the proposed algorithm.

\section{EXPERIMENTAL RESULTS}

In this paper, the proposed method is implemented on HM-11.0 and the results are obtained for both the modified HM11.0 and the original one. For each video sequence, the quantization parameters are 32, 38 and 42. Five frames in the test sequence are encoded. Figures 4 and 5 show the PSNR for different bit rates. Figure 6 compares the subjective video quality (better quality can be shown on a screen). 


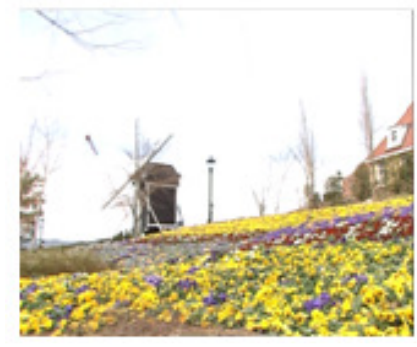

Figure 6: (a) input picture

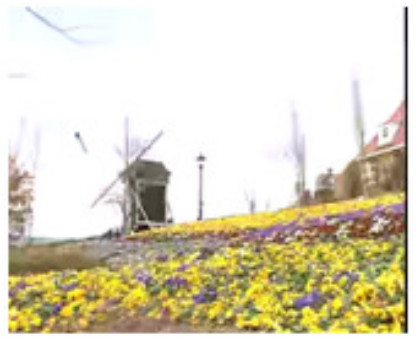

(b) reconstructed picture using original software

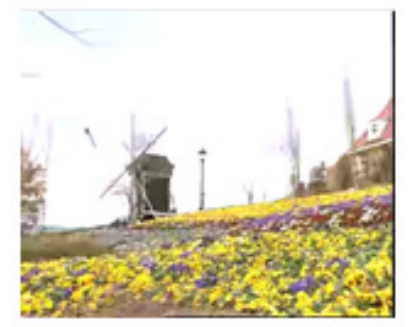

(c) reconstructed picture using proposed software

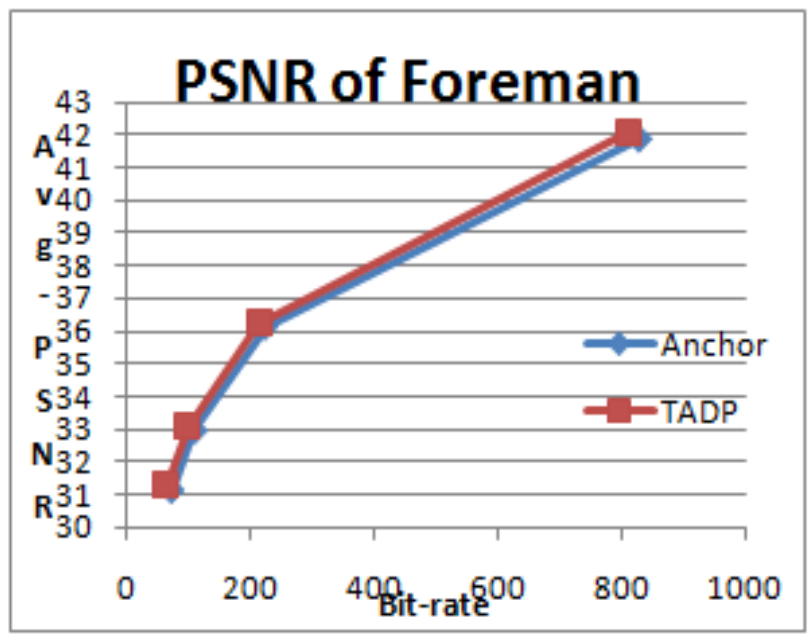

Figure 4:PSNR for different bit rates using Foreman.

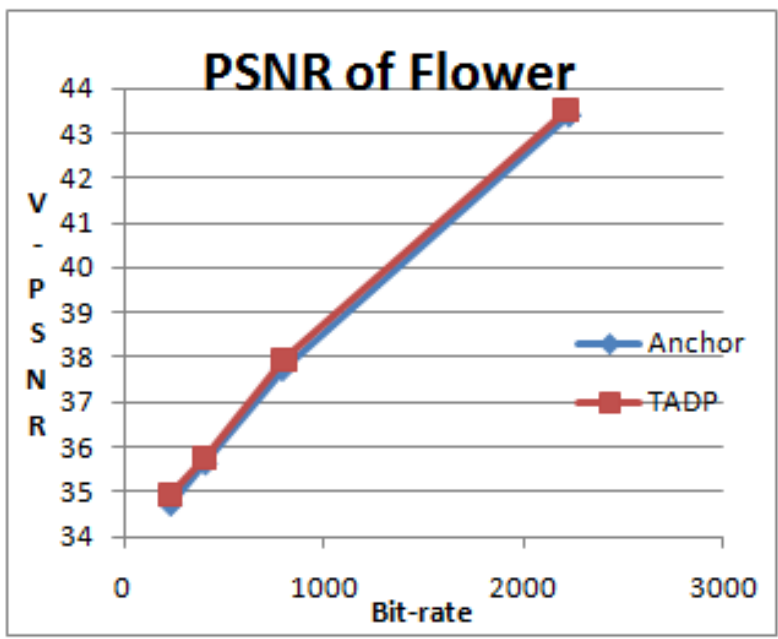

Figure 5: PSNR for different bit rates using Flower. 


\begin{tabular}{|l|l|l|l|l|}
\hline \multicolumn{5}{|c|}{ Table 1: Flower YUV. } \\
\hline QP & $\begin{array}{l}\text { Bitrate } \\
\text { changes \% }\end{array}$ & $\begin{array}{l}\text { Y- } \\
\text { PSNR }\end{array}$ & $\begin{array}{l}\text { U- } \\
\text { PSNR }\end{array}$ & $\begin{array}{l}\text { V- } \\
\text { PSNR }\end{array}$ \\
\hline 32 & 0.69 & 0.03 & 0.10 & 0.18 \\
\hline 38 & 0.66 & 0.05 & 0.21 & 0.27 \\
\hline 42 & 0.65 & 0.07 & 0.34 & 0.35 \\
\hline
\end{tabular}

Table 1: Bit-rate and PSNR changes for test sequence

\begin{tabular}{|l|l|l|l|l|}
\hline \multicolumn{5}{|c|}{ Table 2: Foreman YUV. } \\
\hline QP & $\begin{array}{l}\text { Bitrate } \\
\text { changes } \%\end{array}$ & $\begin{array}{l}\text { Y- } \\
\text { PSNR }\end{array}$ & $\begin{array}{l}\text { U- } \\
\text { PSNR }\end{array}$ & V-PSNR \\
\hline 32 & 0.67 & 0.05 & 0.245 & 0.291 \\
\hline 38 & 0.65 & 0.039 & 0.338 & 0.349 \\
\hline 42 & 0.63 & 0.035 & 0.157 & 0.156 \\
\hline
\end{tabular}

Table 2:Bit-rate and PSNR changes for test sequence

Tables 1 and 2 show that the proposed algorithm reduces the bit rate by $0.7 \%$ and improves the PSNR values by $0.05 \%, 0.30 \%$ and $0.35 \%$ in luminance and chroma. Improvement is more significant on low resolution than the high resolution video sequences.

\section{CONCLUSION}

In this paper, the main aim of proposing a new adaptive trilateral filter for in-loop filtering is to reduce the bit-rate and improve the PSNR values. The simulation results show that the proposed algorithm improves rate distortion performance and reduces the ringing artifacts introduced by the use of large transform block sizes and, therefore, it also improves the perceived video quality. Moreover, proposed algorithm allows an average bitrate reduction of about $0.7 \%$ and improves the PSNR of the decoded frame by $0.05 \%, 0.30 \%$ and $0.35 \%$ in luminance and chroma.

\section{REFERENCES}

[1]. M. T. Pourazad, C. Doutre, M. Azimi, and P. Nasiopoulos“ HEVC: The New Gold Standard for Video Compression,” IEEE consumer electronics magazine, July 2012.

[2]. R. Palaparthi, V. K. Srivastava "A Simple Deblocking Method for Reduction of Blocking Artifacts," IEEE Students' Conference on Electrical, Electronics and Computer Science, 2012.

[3]. X. Zhang, C. R. Xiong and S. Ma, "Adaptive Loop Filter with Temporal Prediction," 2012 Picture Coding Symposium, May 7 - 9, 2012, Kraków, Poland.

[4]. K. Q. Dinh and H. Shim"DEBLOCKING FILTER FOR ARTIFACT REDUCTION IN DISTRIBUTED COMPRESSIVE VIDEO SENSING”VCIP,page 1-5,IEEE(2012)

[5]. M. Naccari and F. Pereira, Instituto de Telecomunicações "Adaptive Bilateral Filter for Improved InLoop Filtering in the Emerging High Efficiency Video Coding Standard," 2012 Picture Coding Symposium May 7-9, 2012, Kraków, Poland.

[6]. C. Y. Tsai, Member, IEEE, C. Y. Chen, T. Yamakage“Adaptive loop filter for video coding."IEEE Journal of selected topics in signal processing Vol.7 N0. 6 ,December 2013.

[7]. S. Esenlik, M. Narroschke, and T.Wedi, "Syntax refinements for SAO and ALF,", Joint Collaborative Team on Video Coding (JCT-VC) of ISO/IEC MPEG and ITU-T VCEG, JCTVC-G566, Nov. 2011..

[8]. http://www.researchgate.net/publication/37988010_Fast_Trilateral_Filtering/file/d912f50d172f2 a9ecf.pdf

[9]. http://www.apsipa.org/proceedings_2012/papers/102.pdf. 